ISSN: $1130-3743$ - e-ISSN: 2386-5660

DOI: http://dx.doi.org/10.14201/teoredu201729179100

\title{
EDUCACIÓN PARA LA SOSTENIBILIDAD Y EDUCACIÓN EN DERECHOS HUMANOS: DOS CAMPOS QUE DEBEN VINCULARSE
}

\author{
Education for Sustainability and Education for Human \\ Rights: two domains that must be linked
}

\section{Éducation à la Durabilité et Éducation aux Droits \\ Humains: deux domaines qui doivent être associés}

\section{Daniel GIL PÉREZ y Amparo VILCHES}

Universitat de València. Facultad de Magisterio.

Departamento de Didáctica de las Ciencias Experimentales y Sociales.

Avenida Tarongers,4.46022 Valencia.daniel.gil@uv.es; amparo.vilches@uv.es

Fecha de recepción: enero de 2017

Fecha de aceptación: marzo de 2017

RESUMEN

En el campo de la educación nos encontramos actualmente con dos importantes requerimientos, sólidamente fundamentados, para introducir la Educación para la Sostenibilidad y la Educación en Derechos Humanos. Se trata, en efecto, de dos áreas educativas prioritarias, a las que UNESCO ha dedicado sendos decenios. Es preciso reconocer, sin embargo, la escasa conexión entre ambas, así como su insuficiente presencia en el sistema educativo, muy particularmente en el nivel universitario. Nuestro propósito en este trabajo es, precisamente, mostrar que, contrariamente a lo que, en general, se ha venido considerando, ambos campos de conocimiento y actividad social están estrechamente vinculados y deben ser abordados conjuntamente para favorecer su mutua potenciación y contribuir a su mayor efectividad. Una Educación para la Sostenibilidad y Derechos Humanos puede convertirse así en 
un factor clave para la necesaria transición a sociedades sostenibles y un elemento esencial de la nueva Ciencia de la Sostenibilidad.

Palabras clave: Educación para la Sostenibilidad; Educación en Derechos Humanos; Ciencia de la Sostenibilidad; Transición a la Sostenibilidad; Educación Superior.

\section{SUMMARY}

There are nowadays in Education two important and well founded claims to introduce Education for Sustainability and Education for Human Rights. In fact UNESCO considers both of them as priority domains and has dedicated a decade to each one. We have to regret, nonetheless, the scarcity of links between both domains, as well as their insufficient presence in the educational system, particularly at the university level. The aim of this paper is, very precisely, to show that, against what has usually been considered, both domains of knowledge and social activity are deeply linked and have to be approached ensemble to favour their mutual enhancement and contribute to a higher efficiency. An Education for Sustainability and Human Rights can become a key contribution to the necessary transition to sustainable societies and an essential element of the new Sustainability Science.

Key words: Education for Sustainability; Education for Human Rights; Sustainability Science; Transition to Sustainability; Higher Education.

\section{SOMMAIRE}

Dans le champ de l'éducation on trouve maintenant deux importantes et bien fondées demandes pour l'introduction de l'Éducation à la Durabilité et de l'Éducation aux Droits de l'Homme. Il s'agit, effectivement, de deux domaines éducatifs prioritaires, et UNESCO a dédié une décennie à chacun d'eux. Il faut regretter, néanmoins, le manque de connexion entre ces deux domaines, ainsi que leur insuffisante présence dans le système éducatif. L'objectif de cet article est, précisément, montrer que, contrairement à ce que, en général, on a considéré jusqu'au présent, ceux deux domaines de connaissance et d'activité social ont des rapports très étroits et doivent être travaillés ensemble pour qu'ils se favorisent mutuellement et deviennent plus efficients. Une Éducation à la Durabilité et aux Droits Humains peut, ainsi, devenir un facteur clé pour la nécessaire transition à des sociétés durables et un élément essentiel de la nouvelle Science de la Durabilité.

Mots clés: Éducation à la Durabilité; Éducation aux Droits de l'Homme; Éducation aux Droits Humains; Science de la Durabilité; Transition à des sociétés durables; Enseignement supérieure. 


\section{INTRODUCCIÓN}

Una abundante literatura y numerosas resoluciones de organismos internacionales vienen insistiendo en la necesidad de introducir la Educación para la Sostenibilidad ${ }^{1}$ en todos los niveles educativos y muy particularmente en la Universidad. Prueba de ello es el papel concedido a la educación en la construcción de un futuro sostenible, tanto por UNESCO como por Naciones Unidas, con iniciativas como el Decenio de la Educación para el Desarrollo Sostenible (DEDS) 2005-2014, que ha dado paso, en 2015, al Programa de Acción Mundial de Educación para el Desarrollo Sostenible (UNESCO, 2014). Estas iniciativas constituyen una clara muestra de la creciente importancia concedida a la Educación para la Sostenibilidad por instituciones internacionales y también nacionales como, por citar un ejemplo destinado específicamente al nivel universitario, la constitución en 2002 del grupo de trabajo para la "Calidad Ambiental y el Desarrollo Sostenible» dentro de la Conferencia de Rectores de las Universidades Españolas.

Sin embargo, pese a este reconocimiento de la importancia de la educación como instrumento para la transición a la Sostenibilidad, los avances en su introducción en el sistema educativo han sido, hasta aquí, claramente insuficientes, como muestran diversos estudios que recogen tanto los avances como las carencias a cubrir (Thomas, 2004; Aznar y Ull, 2009; Barrón Ruiz, Navarrete y Ferrer, 2010; Aznar et al., 2011; Disterheft et al., 2013; Novo y Murga-Menoyo, 2015).

Por nuestra parte, queremos destacar un hecho que hasta aquí no ha recibido suficiente atención entre quienes investigamos y enseñamos en torno a la problemática de la Sostenibilidad, pero que nos parece de la mayor importancia: la situación de la Educación para la Sostenibilidad que acabamos de resumir es muy similar a la relativa a la Educación en Derechos Humanos (DD. HH.), tanto en lo que se refiere a la relevancia atribuida a esta dimensión de la educación, como en lo que respecta a la insuficiencia de su implementación curricular y a los resultados obtenidos. En efecto, como recuerda María de la Paz Pando Ballesteros:

La Declaración formulada por el Consejo de Europa en Estrasburgo, en junio de 2006, bajo el título «Responsabilidad de la Educación Superior para una cultura democrática: Ciudadanía, Derechos Humanos y Sostenibilidad,, manifestaba la convicción de que «la Enseñanza Superior juega un papel esencial en la transmisión de la cultura democrática» y añadía que «la educación superior debe [...] contribuir a la educación de un ciudadano comprometido con la construcción de la paz, la defensa de los derechos humanos y los valores de la democracia” (Pando Ballesteros, 2016a, 65).

1. Sostenibilidad con mayúscula para referirnos al concepto introducido por la comunidad científica y distinguirlo del uso frecuente de las palabras sostenibilidad y sostenible como simples eslóganes, con significados a menudo alejados e incluso contrapuestos a dicho concepto, lo que ha llevado a Robert ENGELMAN (2013) a afirmar que «Vivimos actualmente en una era de la sosteniblabla». 
Un indicador aún más claro de la importancia concedida a esta educación es el hecho de que, como también nos recuerda Pando Ballesteros, «el 10 de diciembre de 2004 la Asamblea General de Naciones Unidas proclamó el "Programa Mundial para la Educación en Derechos Humanos" que está vigente desde 2005 y que se ha desarrollado en tres etapas [...] el Programa Mundial se encuentra en su tercera etapa, a desarrollar entre 2015-2019" (Pando Ballesteros, 2016a, 69).

Pandos reconoce, sin embargo, que, pese a la importancia concedida a esta educación en DD. HH., los avances son muy insuficientes. Señala al respecto, entre otros argumentos, que «En España, la preocupación por articular una educación reglada en derechos humanos, débil y tardía, se ha centrado fundamentalmente en los niveles preuniversitarios, pese a que la universidad es por excelencia una institución formadora de ciudadanos/as, esto es, personas que deben ser capaces de reconocerse como sujetos de derechos» (Pando Ballesteros, 2016a, 65). La situación es, pues, muy similar a la que hemos descrito para la Educación para la Sostenibilidad.

Nos encontramos, en definitiva, con dos importantes requerimientos, sólidamente fundamentados, para introducir tanto la Educación para la Sostenibilidad como la Educación en DD. HH., así como con un similar reconocimiento de su insuficiente presencia, muy en particular en el nivel universitario. Cabe preguntarse, ahora, hasta qué punto este paralelismo es puramente fortuito o tiene un sentido más profundo de vinculación entre ambos campos. Nuestro propósito en este trabajo es, precisamente, mostrar que, contrariamente a lo que en general se ha venido considerando, ambos campos de conocimiento y actividad social están estrechamente vinculados y deben ser abordados conjuntamente para lograr su mutua potenciación y contribuir a su mayor efectividad.

\section{SOSTENIBILIDAD y DERECHOS Humanos: ¿CAMPOS INCONEXOS O VINCULADOS ENTRE Sí?}

Pese al paralelismo que acabamos de mostrar entre Educación para la Sostenibilidad y Educación en DD. HH., se podría pensar que estamos ante dos necesidades educativas importantes, pero inconexas, que habrían de competir entre sí para lograr una atención, hasta aquí insuficiente en ambos casos. Esta visión de desarrollo autónomo se ha visto reforzada por la escasa relación que la literatura ha mostrado, hasta recientemente, entre ambos campos de estudio: las publicaciones que se han ocupado de la problemática de la Sostenibilidad no se han referido, en general, a los DD. HH. y viceversa. Más aún, hablar de la posible vinculación entre Sostenibilidad y DD. HH. ha distado mucho de ser aceptada con facilidad, porque para muchos ciudadanos, e incluso expertos, los DD. HH. eran algo que solo asociaban con cuestiones como el rechazo de la tortura o la reivindicación de libertades frente a las dictaduras y les parecía que se trivializaba cuando se relacionaban con problemas como los relativos al medio ambiente (Vilches, Macías y Gil Pérez, 2014, capítulo 11).

Podemos recordar a este respecto, a título de ejemplo, algunas de las intervenciones de expertos que se produjeron en el encuentro «Diálogo Intercultural sobre 
Democracia y Derechos Humanos", que tuvo lugar en Santiago de Compostela en el año 2000, promovido por unEsco y el periódico El País (Vilches y Gil Pérez, 2003, capítulo 15):

El Comisario Europeo por los DD. HH., a la sazón Álvaro Gil Robles, afirmaba: "Hace unos meses estuve en Chechenia y nadie me hablaba del medio ambiente, sino de salvar su vida, de que no violasen a sus hijas y de que no torturasen a sus hijos». Y Adam Michnick, director de la revista polaca Gazeta Wyborcza, afirmaba aún más contundentemente: «Los derechos humanos no son una receta para todos los dolores de la humanidad. Son solo una propuesta para evitar la tiranía política». Este reduccionismo de los DD. HH. a temas políticos queda reflejado en los medios de comunicación y también, hasta muy recientemente, en los propios informes de la organización mundial de defensa de los DD. HH., Amnistía Internacional.

La situación, sin embargo, ha comenzado a cambiar: Amnistía Internacional inició, en la 25. ${ }^{a}$ reunión de su Consejo Internacional, que tuvo lugar en Dakar en 2001, una profunda revolución en la dirección de superar el reduccionismo de su atención a los derechos políticos. En esa reunión, su presidente Pierre Sané expresó la voluntad de Amnistía Internacional de incluir la denuncia de las desigualdades económicas en la lucha por los DD. HH., porque se había comprendido que la prisión de la pobreza física y mental puede aislar y ser tan cruel como cualquier gulag político. «Esto significa -afirmó Sané- ampliar nuestro objetivo para proteger no solo los derechos civiles y políticos, sino todos los derechos humanos».

Esta falta de vinculación entre DD. HH. y problemática socioambiental la hemos encontrado también al plantear, en 2016, la siguiente pregunta a 20 colegas del área de Didáctica de las Ciencias de varias universidades españolas (Alicante, Granada, Complutense de Madrid, Sevilla y Valencia):

¿En qué medida dirías que gozas de los derechos humanos (en el sentido de sentirte beneficiario de los mismos)? Puedes indicar, si ha lugar, de qué derechos te consideras plenamente beneficiario y de cuáles no y puedes escribir las reflexiones que consideres útiles para expresar tus puntos de vista, dudas, previsiones, etc.

Las respuestas se centraron, mayoritariamente, en los derechos incluidos en la Declaración Universal de los Derechos Humanos, aprobada por Naciones Unidas en 1948, sin incluir, pues, el derecho a un ambiente saludable, ni referirse a la vinculación de los derechos contemplados con la problemática socioambiental.

Sin embargo, la situación a este respecto está cambiando rápidamente, como explican Lívia Gaigher Bósio Campello y Luciani Coimbra de Carvalho: «El estudio de la conexión entre la protección ambiental y los derechos humanos ganó enorme relevancia [...] a medida que la comunidad científica fue adquiriendo conciencia de la incidencia de la degradación y modificación del medio ambiente en el pleno disfrute de los derechos y libertades de los seres humanos", añadiendo que ello ha dado lugar a «un proceso de movilización y redimensionamiento de los derechos humanos globalmente existentes a partir de la consideración de la compleja relación de interdependencia del hombre con el medio ambiente» (Gaigher Bósio Campello y 
Coimbra de Carvalho, 2016, 625-626). Este redimensionamiento supone una ampliación de los derechos ya reconocidos por la comunidad internacional (aunque ello no significa, lamentablemente, que sean plenamente respetados); por ejemplo, «el derecho a la vida se expande e incluye el derecho a vivir en un ambiente saludable, libre de contaminación» (p. 632). Pero, "dado que los derechos humanos existentes fueron formulados en una época en que la preocupación por el medio ambiente era escasa, su capacidad para incluir la problemática ambiental es limitada y se ve la necesidad de incorporar nuevos derechos que la recojan explícitamente» (p. 634).

El resultado de este proceso de ampliación de DD. HH. queda reflejado hoy, por ejemplo, en la incorporación de epígrafes como "Cambio climático», en la "Lista de Temas de Derechos Humanos» que UnESCO ofrece en su sitio oficial. Y el mismo "Programa Mundial para la Educación en Derechos Humanos", instituido por Naciones Unidas en 2005, incluye entre sus fines "favorecer un Desarrollo Sostenible centrado en las personas y la justicia social».

Podemos concluir, pues, que entre quienes se ocupan de DD. HH. se ha ido abriendo la comprensión de la necesidad de tener en cuenta su vinculación con la problemática ambiental y del Desarrollo Sostenible. Cabe ahora preguntarse si quienes trabajan en el campo de la transición a la Sostenibilidad comienzan a atender igualmente a esta vinculación.

La respuesta es positiva, como puede constatarse en el sitio que UNESCO dedica a la Educación para el Desarrollo Sostenible (EDS): «Educar para el Desarrollo Sostenible significa incorporar los temas fundamentales del Desarrollo Sostenible a la enseñanza y el aprendizaje, por ejemplo, el cambio climático, la reducción del riesgo de desastres, la biodiversidad, la reducción de la pobreza y el consumo sostenible». Se mencionan, en estas pocas líneas, tan solo algunos ejemplos de los problemas más graves a los que la humanidad ha de hacer frente, según UNESCO, para hacer posible un Desarrollo Sostenible, pero conviene notar que, entre ellos, figura el problema de la pobreza, vinculando así la problemática ambiental con la social, lo que nos remite, implícitamente, a la problemática de los DD. HH.

Esta vinculación entre la problemática ambiental y social aparece aún más claramente cuando, en vez de mencionar solo algunos ejemplos, se procede a considerar el conjunto de problemas que afectan a la Sostenibilidad. En la Tabla 1 presentamos una síntesis que hemos elaborado como fruto de una doble estrategia de resultados convergentes (Vilches y Gil Pérez, 2003). Por una parte, hemos tomado en consideración, inicialmente, los problemas señalados por la Comisión Mundial del Medio Ambiente y del Desarrollo (1988), completándolos con los contemplados por otros expertos, mediante una amplia búsqueda bibliográfica que hemos mantenido a lo largo de los años (Meadows et al., 1972; Bybee, 1991; Tilbury, 1995; Folch, 1998; Sen, 2000; Mayor Zaragoza, 2000; Diamond, 2006; Novo, 2006; Pearce, 2007; Sachs, 2008; Flavin, 2008; Rifkin, 2010; Bermejo, 2011; Felber, 2012; Klein, 2015; wwF, 2016), con una particular atención a los anuarios La situación del mundo del Worldwatch Institute (1984-2016), elaborados por un amplísimo conjunto de expertos. 
Una segunda estrategia ha consistido en organizar talleres diseñados para favorecer la reflexión colectiva de distintos grupos tanto de docentes -empezando por nuestro propio equipo- como de estudiantes universitarios y de secundaria superior, estructurados en equipos de 4 a 6 miembros (Gil Pérez y Vilches, 2005). Como punto de partida de estos talleres nos planteamos, y propusimos a los demás equipos, la siguiente tarea: "Enumerar los problemas y desafíos a los que, en su opinión, la humanidad ha de hacer frente para encarar su supervivencia. Con esta reflexión colectiva perseguimos comenzar a construir una visión lo más completa y correcta posible de la situación existente y de las medidas a adoptar al respecto».

Cabe señalar que cuando se pide una reflexión individual similar a la que plantea esta actividad, se obtienen, en general, visiones muy fragmentarias, a menudo centradas casi exclusivamente en los problemas de contaminación ambiental y agotamiento de recursos, con olvido de muchos otros aspectos igualmente relevantes. Si, por el contrario, se propone esta tarea a los equipos, los resultados de cada equipo son bastante más positivos y la puesta en común de sus contribuciones cubre la práctica totalidad de los aspectos considerados por los expertos. Los problemas menos mencionados por los equipos son el de la urbanización desordenada y especulativa, la destrucción de la diversidad cultural y el crecimiento demográfico, pero las puestas en común permiten salir al paso de estos frecuentes olvidos.

TABLA 1

PROBLEMAS Y DESAFÍOS A LOS QUE DEBE HACER FRENTE LA HUMANIDAD PARA HACER POSIBLE UN PRESENTE Y UN FUTURO SOSTENIBLES

1. Poner fin a un crecimiento económico y demográfico que resulta agresivo con el medio físico y nocivo para los seres vivos, fruto de comportamientos guiados por intereses y valores particulares y a corto plazo.

Dicho crecimiento se traduce en una serie de problemas específicos pero estrechamente relacionados:

1.1. Una urbanización creciente y, a menudo, desordenada y especulativa, acompañada del abandono y deterioro del mundo rural.

1.2. Contaminación ambiental pluriforme y sin fronteras con graves secuelas "glocales» (a la vez locales y globales): lluvia ácida, destrucción de la capa de ozono, incremento del efecto invernadero (causa principal del cambio climático), etc.

1.3. Agotamiento y destrucción de los recursos (yacimientos minerales, capa fértil de los suelos, recursos de agua dulce, etc.).

1.4. Degradación de ecosistemas (incluidos los espacios humanizados), destrucción de la biodiversidad (causa de enfermedades, hambrunas...) y, en última instancia, desertificación.

1.5.Destrucción, en particular, de la diversidad cultural. 
2. Poner fin a las siguientes causas -o, si se prefiere, nuevos problemas-de este crecimiento no sostenible:

2.1. El hiperconsumo de las sociedades «desarrolladas» y grupos poderosos.

2.2. La explosión demográfica en un planeta de recursos limitados.

2.3. Los desequilibrios existentes entre distintos grupos humanos -asociados a falta de libertades e imposición de valores e intereses particulares- que se traducen en hambre, pobreza, discriminación étnica, de género... y, en general, marginación de amplios sectores de la población.

2.4. Las distintas formas de conflictos y violencias asociados, a menudo, a dichos desequilibrios:

2.4.1. Las violencias de clase, género, interétnicas, interculturales... y los conflictos bélicos (con sus secuelas de carrera armamentística, destrucción...).

2.4.2. La actividad de las organizaciones mafiosas que trafican con armas, drogas y personas, contribuyendo decisivamente a la violencia ciudadana.

2.4.3. La actividad especuladora y depredadora de empresas transnacionales que escapan al control democrático imponiendo condiciones de explotación destructivas de personas y medio físico, eludiendo insolidariamente sus obligaciones tributarias a través de los llamados paraísos fiscales.

NOTA: No se pretende poner énfasis en la distinción entre problemas y causas, que pueden considerarse también como otros tantos problemas, todos ellos estrechamente vinculados.

Somos conscientes, por supuesto, de que no basta con elaborar un listado de problemas, como el que recoge la Tabla 1, para impulsar comportamientos adecuados: en realidad, cualquier ciudadana o ciudadano ha oído hablar reiteradamente, en los medios de comunicación, de problemas como la contaminación, el cambio climático, el agotamiento de recursos fundamentales, la pérdida de biodiversidad, el hambre de millones de personas, etc. Sin embargo, este conocimiento no supone que se sea consciente de la gravedad de la situación y de la necesidad de actuar con urgencia.

Se puede comprender esta aparente incoherencia si se analiza la manera inconexa de abordar los problemas en los medios de difusión (e incluso en bastantes trabajos especializados): buscando la novedad de las noticias, la atención pasa rápidamente de, por ejemplo, la destrucción de la capa de ozono al agotamiento del petróleo, para saltar al cambio climático y de ahí al problema de la falta de agua, o a la pobreza extrema de miles de millones de seres humanos... Cada problema es desplazado por uno nuevo y el resultado es que ninguno de ellos llega a ser visto como demasiado preocupante, puesto que siempre hay otro que viene a sustituirlo en el palmarés de las urgencias (Vilches, Macías y Gil Pérez, 2014).

La aparente "competencia" entre los problemas -que se traduce en la mutua neutralización de la atención que concitan- es el fruto del tratamiento inconexo de cada uno de ellos, sin atender a su estrecha vinculación como aspectos que se 
potencian mutuamente de una misma problemática y que deben abordarse, pues, conjuntamente. Esta vinculación aparece con nitidez a poco que se profundice en los problemas, y así sucede en los talleres que organizamos. Cuando se analiza el agotamiento de recursos como, por ejemplo, la masa boscosa, se comprende que su reducción no es solo debida a una tala excesiva, sino que la lluvia ácida, la contaminación, juega un papel nada despreciable. Y esa desaparición de la masa boscosa, como sumidero del dióxido de carbono, contribuye, a su vez, al cambio climático. Estos dos problemas de contaminación y agotamiento de recursos se vinculan, a su vez, con el de una urbanización creciente y desordenada: los informes elaborados para Naciones Unidas por paneles de expertos alertan, año tras año, de un deterioro generalizado de los ecosistemas que califican de devastador y que atribuyen tanto a la explotación intensiva de recursos como a la contaminación, a la urbanización desordenada, a los incendios, etc. Y la acción concertada de estos problemas no solo está destruyendo todos los ecosistemas -bosques, praderas, humedales, arrecifes de coral...-, sino que contribuye al cambio climático, al agotamiento de recursos esenciales, a una grave pérdida de biodiversidad, a la desertización y, en consecuencia, a la pobreza extrema y al hambre de extensas poblaciones.

Es importante, pues, comprender que estos problemas socioambientales están estrechamente vinculados entre sí y se potencian mutuamente, por lo que resulta imposible resolver ninguno de ellos sin tener en cuenta los restantes (Duarte, 2006). En ello insiste Jared Diamond, en su libro Colapso, tras analizar 12 grupos de problemas a los que se enfrentan nuestras sociedades: «Si no resolvemos cualquiera de la docena de problemas sufriremos graves perjuicios [...] porque todos ellos se influyen mutuamente» (Diamond, 2006, 645).

Se llega así a comprender que la contaminación, el cambio climático, el agotamiento de recursos esenciales, etc., están vinculados a los desequilibrios, a la pobreza, al hambre..., es decir, a la misma problemática de los DD. HH. En el mismo sentido, Amartya Sen (2000), en su libro Desarrollo y Libertad, concibe la expansión de las libertades como un pilar fundamental para abordar la problemática de la Sostenibilidad, como elemento clave para la lucha contra el hambre y la pobreza. Una pobreza que significa malnutrición, reducción de la esperanza de vida, falta de acceso a agua potable y condiciones de salubridad, enfermedades, imposibilidad de acceder a la escuela, a la cultura... (Cortina y Pereira, 2009).

Intentaremos seguidamente profundizar en esta vinculación y, en consecuencia, en la necesidad del pleno reconocimiento y respeto de los DD. HH. para hacer posible la transición a la Sostenibilidad y, simétricamente, en la necesidad de la transición a la Sostenibilidad para avanzar en el respeto y universalización de los DD. HH.

\section{LA UNIVERSALIZACIÓN DE LOS DERECHOS HUMANOS Y LA TRANSICIÓN A LA SOSTENIBILIDAD SE NECESITAN Y POTENCIAN MUTUAMENTE}

Abordaremos, en primer lugar, el papel de los DD. HH. en la transición a la Sostenibilidad y, en una segunda sección, estudiaremos cómo la transición 
a la Sostenibilidad influye en la universalización de los DD. HH. Para mejor comprender esta estrecha vinculación, intentaremos salir al paso de algunas concepciones erróneas, acerca de ambos conceptos, que constituyen serios obstáculos para dicha comprensión y para una acción eficaz.

\subsection{Papel de los Derechos Humanos en la transición a la Sostenibilidad}

Empezando por los DD. HH., M. ${ }^{\text {a }}$ Esther Martínez Quinteiro señala que «Todo el mundo parece saber qué se quiere decir cuando se habla de derechos humanos o cuando se denuncia su transgresión, y, sin embargo, el "discurso de los derechos humanos" arrastra desde su nacimiento el "síndrome de la Torre de Babel": no hay, no ha habido, un concepto y un discurso único sobre los derechos humanos" (Martínez Quinteiro, 2016, 41). Y no lo ha habido, entre otras razones, porque se trata de un concepto surgido recientemente y que sigue en construcción. Así nos lo recuerda Pando Ballesteros: "Son muchos y prestigiosos los especialistas que reconocen actualmente la historicidad del discurso de los derechos humanos y su carácter evolutivo, acumulativo y progresivo, no necesariamente lineal» (Pando Ballesteros, 2016b, 17). En ello insiste también Martínez Quinteiro: «Solo es esencialmente antihistoricista el discurso sobre los derechos humanos producido por el iusnaturalismo religioso, en cuanto supone que es Dios el supuesto creador de un derecho natural intemporal" (Martínez Quinteiro, 2016, 42). Esta visión intemporal, ahistórica, es sostenida, por ejemplo, por José do Nascimento, quien escribe: "Cuando hablamos de "principios de los derechos humanos" podemos afirmar, sin sombra de duda, que están presentes desde el inicio de la creación» (Nascimento, 2016, 99). Esta ausencia de dudas es ya suficientemente preocupante desde el punto de vista científico y a ello se añade la falta de coherencia de este punto de vista con los hechos históricos, bien establecidos, acerca del surgimiento y desarrollo de los DD. HH. Una falta de coherencia que afecta al pensamiento y actuación de muchos seguidores de distintas religiones, que ni siquiera reconocen la plena igualdad de derechos entre hombres y mujeres. "La evidencia, con todo -afirma Martínez Quinteiro (2016, 42)- llevará a muchos iusnaturalistas religiosos a matizar este antihistoricismo ontológico o esencialista con la afirmación de la historicidad del reconocimiento o "descubrimiento" de los derechos humanos por los hombres".

Podemos concluir, pues, que el análisis histórico de las referencias a los DD. HH. no permite hablar de su "existencia» intemporal, como parte de un "derecho natural", sino que obliga a aceptar el surgimiento histórico de una opción ética y política que vino a defender, contra concepciones y prácticas inveteradas, que todos los seres humanos hemos de tener unos mismos derechos fundamentales. Un surgimiento, por lo demás, conflictivo (De la Rosa, 2015), que se ha enfrentado -y sigue enfrentándose- a tradiciones y comportamientos muy enraizados de «defensa de nuestro bienestar contra los demás» que se traducen en falta de equidad y desequilibrios insostenibles. Comportamientos que han hecho posible, durante milenios, la esclavitud de los vencidos y toda suerte de discriminaciones, por razones 
de género, étnicas, de clase social, etc., en buena parte vigentes todavía en unos $\mathrm{u}$ otros lugares del planeta.

Es necesario, además, distinguir entre la reivindicación que un colectivo puede hacer de determinados derechos para sus miembros y la propuesta de extender unos derechos considerados fundamentales a toda la especie humana. Solo en este último caso de planteamiento universalista cabe hablar de DD. HH.: "La Declaración Universal de los Derechos Humanos es la primera constitución de la Historia que nace con la pretensión explícita de abarcar a toda la humanidad» (De la Rosa, 2015, 10). No debe olvidarse, a este respecto, que los "Droits de l'Homme" de la Revolución francesa, por citar un ejemplo ilustre, excluían explícitamente a las mujeres (ique solo consiguieron el derecho al voto en Francia tras la Segunda Guerra Mundial!), ni que la desigualdad social de las mujeres se oponga, todavía hoy, a la realización efectiva de los DD. HH.: "Cambiar la situación social de las mujeres es un requisito para que los DD. HH. sean una realidad no solo para las mujeres sino para la especie humana" (Izquierdo Benito, 2015, 42). En efecto, nadie puede disfrutar plenamente de derechos, concebidos como universales, mientras millones de seres humanos quedan discriminados.

Tampoco debe olvidarse que el contenido de estos derechos sigue evolucionando. Así lo expresa, por ejemplo, Norberto Bobbio, citado por Pando Ballesteros (2016b, 17): "La lista de los derechos se ha modificado y sigue haciéndolo con el cambio de las condiciones históricas, esto es, las necesidades, los intereses, el poder, etc.». Ni siquiera podemos decir que exista una única lista de los derechos en cada momento, puesto que la realidad es bastante más compleja y se debe distinguir entre las reivindicaciones iniciales y el reconocimiento legal de los derechos como jurídicamente vinculantes, a lo largo de un proceso confuso e -insistimos- conflictivo, durante el cual las distintas reivindicaciones pueden ir cobrando fuerza en uno u otro lugar, hasta llegar, en algunos casos, a formar parte del «discurso internacional sobre los derechos humanos» y ser reconocidos como jurídicamente vinculantes por los signatarios de los correspondientes acuerdos internacionales (Martínez Quinteiro, 2016, 53). Es así como se ha llegado a consensuar la reivindicación de tres generaciones de DD. HH. (Vercher, 1998), cuya vinculación con la Sostenibilidad aparece hoy con claridad (Vilches, Macías y Gil Pérez, 2014, capítulo 11):

- Derechos democráticos, civiles y políticos (de opinión, reunión, asociación...) para todas y todos, sin limitaciones de origen étnico, de género, etc., que constituyen la primera generación de DD. HH.

Como puede comprenderse, estos derechos constituyen una condición sine qua non para la participación ciudadana en la toma de decisiones que afectan al presente y futuro de la sociedad (Sen, 2000) y, por tanto, para la transición a la Sostenibilidad. Pensemos, por ejemplo, en la importancia de una información adecuada de los riesgos ambientales, para una toma de decisiones fundamentada (Gaigher Bósio Campello y Coimbra de Carvalho, 2016, 634). Ello exige el pleno reconocimiento no solo del derecho a la libertad de opinión y expresión, sino 
incorporar también el «derecho fundamental de los ciudadanos a recibir información veraz» (Almuiña, 2016, 170).

- Derechos económicos, sociales y culturales, o "Derechos humanos de segunda generación", que incluyen hoy, como sabemos, el derecho a la salud; a una alimentación adecuada; a un trabajo satisfactorio; a una vivienda en un entorno saludable; a una educación de calidad a lo largo de toda la vida; a la cultura como eje vertebrador de un desarrollo personal y colectivo estimulante y enriquecedor; a la planificación familiar y al libre disfrute de la sexualidad, siempre que no conculque la libertad de otras personas; etc.

Estos derechos aparecen como un requisito y, a la vez, como un objetivo del Desarrollo Sostenible. ¿Se puede exigir a alguien, por ejemplo, que no contribuya a esquilmar un banco de pesca si ese es su único recurso para alimentar a su familia? No es concebible tampoco, por citar otro ejemplo, la interrupción de la explosión demográfica -que está llevando a superar la capacidad de carga del planeta- sin el reconocimiento del derecho a la planificación familiar y al libre disfrute de la sexualidad no necesariamente vinculada a la reproducción. Y ello remite, a su vez, al derecho a la educación.

Como puede constatarse, estas dos generaciones de derechos, "si son plenamente movilizados y redimensionados, pueden ofrecer una gran contribución a la protección del medio ambiente global» (Gaigher Bósio Campello y Coimbra de Carvalho, 2016, 634). No basta, sin embargo, con esta contribución a la Sostenibilidad de los DD. HH. contemplados por ambas generaciones, porque, como estas mismas autoras señalan, estos derechos fueron concebidos en épocas en que apenas había preocupación por el medio ambiente. Y añaden un segundo e importante argumento: "Las democracias son enteramente capaces de destrucción ambiental y están estructuralmente predispuestas para el consumo ilimitado. Las democracias industriales de los países desarrollados, con sus sistemas legales basados en derechos liberales, son desproporcionadamente responsables de gran parte de los daños ambientales, incluyendo el consumo excesivo de recursos finitos y las emisiones de gases de efecto invernadero" (Gaigher Bósio Campello y Coimbra de Carvalho, 2016, 635-636). Se ha visto, por ello, la necesidad de incorporar derechos vinculados explícitamente con la Sostenibilidad:

- Derechos Humanos de Tercera Generación, denominados «derechos ambientales» por Gaigher Bósio Campello y Coimbra de Carvalho (2016, 634) y "derechos de solidaridad» por Vercher (1998). Incluyen, de forma destacada, el derecho a un ambiente saludable, a la paz y al Desarrollo Sostenible para todos los pueblos y para las generaciones futuras.

Como afirma Vercher (1998), la incorporación del derecho al medio ambiente saludable como un derecho humano, esencialmente universal, responde a un hecho incuestionable: "De continuar degradándose el medio ambiente al paso que 
va degradándose en la actualidad, llegará un momento en que su mantenimiento constituirá la más elemental cuestión de supervivencia en cualquier lugar y para todo el mundo [...] El problema radica en que cuanto más tarde en reconocerse esa situación mayor nivel de sacrificio habrá que afrontar y mayores dificultades habrá que superar para lograr una adecuada recuperación».

Por lo que respecta al derecho a la paz, responde a la necesidad de impedir que los intereses particulares (económicos, culturales...) se impongan por la fuerza a los demás, con grave perjuicio para todos; recordemos las consecuencias de los conflictos bélicos y de la simple preparación de los mismos: desde la degradación ambiental (no hay nada tan contaminante y destructor de recursos como un conflicto bélico) a los millones de refugiados, víctimas de las guerras (Vilches, Macías y Gil Pérez, 2014, capítulo 11).

Por último, pero no menos importante, esta tercera generación de derechos incluye el derecho a un Desarrollo Sostenible de todos los pueblos. Ello conlleva, por una parte, la superación de los actuales desequilibrios económicos, entre países y poblaciones, así como nuevos modelos y estructuras económicas para la Sostenibilidad, que contribuyan a poner fin al conjunto de problemas interconectados mencionados, y que garanticen, por una parte, un acceso universal sostenible a los recursos básicos (tanto materiales como energéticos limpios y renovables) y, por otra, la defensa de la diversidad cultural, como patrimonio de toda la humanidad, y del mestizaje intercultural, contra todo tipo de racismo y de barreras étnicas o sociales. Se trata de un derecho que, como veremos en el siguiente apartado, exige clarificación, dadas las confusiones y tergiversaciones que el concepto de Desarrollo Sostenible ha originado y sigue provocando.

Este amplio conjunto de derechos son interdependientes y ninguno de ellos puede ser abordado ignorando los demás. No tiene sentido, por ejemplo, pretender satisfacer el derecho a una alimentación adecuada de la población recurriendo a prácticas agrícolas que contribuyan a la degradación ambiental y pongan en peligro el futuro mismo de la especie. Es necesario, pues, abordar las contradicciones que pueden surgir en la satisfacción de diferentes derechos (García, 2015).

Por supuesto, siguen surgiendo nuevas reivindicaciones y se habla ya de una cuarta generación de derechos, asociados a los cambios tecnológicos y, en particular, al entorno digital. Es lo que sostiene Rosa San Segundo, quien afirma: "Las nuevas denominaciones de homo digitalis, bomo connectatus u bomo ciber suponen ya un nuevo concepto y estadio en la era de la humanidad" (San Segundo, 2016, 651). Pero en este trabajo hemos querido limitarnos a considerar el papel que pueden jugar, en la transición a la Sostenibilidad, las tres primeras generaciones de DD. HH., para cuya reivindicación (aunque todavía no para su efectiva aplicación universal) existe ya un amplio consenso.

Naturalmente, la relación entre Sostenibilidad y DD. HH. es biyectiva: si el respeto de los derechos es, como hemos visto, condición indispensable para avanzar en la transición a la Sostenibilidad, esta es necesaria, a su vez, para hacer posible la universalización de los DD. HH. 


\subsection{Papel de la transición a la Sostenibilidad en la universalización de los Derechos Humanos}

También los conceptos de Sostenibilidad y de Desarrollo Sostenible precisan ser clarificados para salir al paso de confusiones y distorsiones (Vilches, Macías y Gil Pérez, 2014, capítulo 1) que constituyen serios obstáculos para avanzar en la transición a la Sostenibilidad y comprender su papel en la universalización de los DD. HH.

Comenzaremos recordando que el concepto de Sostenibilidad surge por vía negativa, como resultado de los análisis de la situación del mundo, que obligó a hablar de "emergencia planetaria", de una situación insostenible, fruto de las actividades humanas, que amenaza gravemente el futuro de la misma humanidad (Bybee, 1991), por acercarse peligrosamente a los límites del planeta e incluso haber superado ya algunos de ellos (Folke, 2013). Se habla incluso de una etapa geológica nueva, el Antropoceno, término propuesto por el premio Nobel Paul Crutzen para destacar la responsabilidad de la especie humana en los profundos cambios que está sufriendo el planeta (Sachs, 2008).

Conviene resumir sucintamente esta situación de insostenibilidad para mejor comprender su estrecha vinculación con la problemática de los DD. HH. (Vilches y Gil Pérez, 2015):

Es insostenible el actual ritmo de utilización de todo tipo de recursos esenciales, desde los energéticos a los bancos de pesca, los bosques, las reservas de agua dulce y el mismo suelo cultivable. Un ritmo muy superior al de su regeneración, cuando son renovables, o al de su sustitución por otros que sí lo sean.

Es insostenible el ritmo de producción de residuos contaminantes, muy superior al de la capacidad del planeta para digerirlos: una contaminación pluriforme y $\sin$ fronteras, que envenena suelos, ríos, mares y aire y afecta ya a todos los ecosistemas.

Es insostenible, en particular, el acelerado incremento de la concentración de gases de efecto invernadero en la atmósfera, de origen claramente antrópico, que está provocando un desarreglo climático visible ya, entre otras muchas consecuencias, en la rápida disminución de las llamadas nieves perpetuas (la más importante reserva de agua dulce con la que cuentan miles de millones de seres humanos) y en el aumento de la frecuencia e intensidad de fenómenos atmosféricos extremos (huracanes, inundaciones, sequías e incendios...); el cambio climático contribuye así a un proceso de degradación generalizada que corre el riesgo de llegar a ser irreversible, haciendo inhabitable la Tierra para la especie humana.

Es insostenible el proceso de urbanización acelerada y desordenada -acompañado del abandono del mundo rural- que potencia los efectos de la contaminación (a causa del transporte, calefacción, acumulación de residuos, etc.) y el agotamiento de recursos (con la destrucción de terrenos agrícolas, el aumento de los tiempos de desplazamiento y consiguiente consumo de recursos energéticos, etc.).

Es insostenible el crecimiento explosivo de la población mundial, que puede estar ya cerca de sobrepasar -si no lo ha hecho ya- la capacidad de carga del planeta: la especie humana acapara ya casi tanta producción fotosintética como la 
totalidad de las restantes especies y su huella ecológica (es decir, el área de territorio ecológicamente productivo necesaria para producir los recursos utilizados y para asimilar los residuos producidos) ha superado ampliamente la biocapacidad del planeta.

Es insostenible la acelerada pérdida de biodiversidad, que obliga a hablar de una sexta gran extinción ya en marcha, que amenaza con romper los equilibrios de la biosfera y arrastrar a la propia especie humana, causante de esta masiva extinción.

Es insostenible igualmente la pérdida de diversidad cultural y, muy en particular, de culturas campesinas milenarias. No debemos olvidar que la diversidad de culturas es la garantía de una pluralidad de respuestas a los problemas a los que ha de hacer frente la humanidad. Cada cultura no es solo una riqueza para el pueblo que la ha creado, sino un patrimonio de toda la humanidad.

Es insostenible e inaceptable el desequilibrio entre una quinta parte de la humanidad impulsada al hiperconsumo y miles de millones de personas que sufren hambre y condiciones de vida insoportables.

Es insostenible, en definitiva, un sistema socioeconómico guiado por la búsqueda del máximo beneficio particular a corto plazo, que apuesta por el crecimiento económico indefinido en un planeta finito, sin atender a sus consecuencias ambientales y sociales, lo que le convierte en responsable de los problemas que acabamos de enumerar y de otros igualmente graves, como las dramáticas migraciones, los conflictos y violencias causados por la competitividad, por el afán de controlar los recursos energéticos y otras materias primas esenciales y, en suma, por una destructiva falta de equidad y anteposición de intereses particulares a la cooperación en beneficio de todos los seres humanos y de las generaciones futuras, amenazando así el presente y el futuro de la humanidad: "Estos preocupantes problemas están causando miseria, dolor y muerte, si bien de forma muy desigual, a la humanidad: mientras unos sufren y mueren, otros miran hacia otro lado, intentando permanecer ajenos a esta cruda realidad, como si no fuéramos todos pasajeros de la nave Tierra y partícipes, por tanto, de un destino común» (García Pérez, 2015, 149).

Un futuro amenazado es, precisamente, el título del primer capítulo de Nuestro futuro común, el informe de la Comisión Mundial del Medio Ambiente y del Desarrollo (1988), a la que debemos uno de los primeros intentos de introducir el concepto de Sostenibilidad: «El Desarrollo Sostenible es el desarrollo que satisface las necesidades de la generación presente sin comprometer la capacidad de las generaciones futuras para satisfacer sus propias necesidades».

Se trata, en opinión formulada, ya en la década de los 90 del siglo pasado, por el biólogo y educador Rodger W. Bybee, de «la idea central unificadora más necesaria en este momento de la historia de la humanidad" (Bybee, 1991), aunque se abre paso con dificultad y ha generado incomprensiones y distorsiones como, muy en particular, la confusión entre desarrollo y crecimiento, que conduce, incluso, a que algunos autores rechacen el concepto de "desarrollo sostenible» como un 
oxímoron, una manipulación de los «desarrollistas», de los partidarios del crecimiento económico continuado, que pretenden hacer creer en la compatibilidad del crecimiento con la sostenibilidad ecológica (Naredo, 1998; García, 2004). La idea de un Desarrollo Sostenible, sin embargo, parte de la distinción entre desarrollo y crecimiento, concibiendo la posibilidad (y necesidad) de mejoras cualitativas o despliegue de potencialidades, sin crecimiento, es decir, sin incremento cuantitativo de la escala física, sin incorporación de mayor cantidad de materiales. Con otras palabras: es el crecimiento lo que no puede continuar indefinidamente en un mundo finito, pero sí es posible el desarrollo. Posible y necesario, porque las actuales formas de vida no pueden continuar, deben experimentar cambios cualitativos profundos, tanto para aquellos (la mayoría) que viven en la precariedad como para el 20\% que practica un consumo depredador, insostenible. Y esos cambios cualitativos suponen un desarrollo sin crecimiento, que será preciso diseñar y orientar adecuadamente, ajustando la economía a las exigencias de la ecología y del bienestar social global, es decir, orientando la economía al logro de la Sostenibilidad, necesaria a su vez para la plena universalización de los DD. HH.

Gaigher Bósio Campello y Coimbra de Carvalho (2016, 628-631) ejemplifican la influencia de la problemática medioambiental sobre los derechos humanos. Señalan así que "como la desaparición de bosques puede potenciar los cambios climáticos, la pérdida de biodiversidad o la protección contra inundaciones, la desforestación puede tener impacto sobre los derechos a la vida y a la salud». Se refieren igualmente al «aumento de la población y a la pobreza [...] Este aumento de la población puede tener un impacto indirecto sobre los derechos humanos debido a las tensiones que pueden provocarse en la protección del derecho a la vida, a la salud, al agua, a la alimentación y a la vivienda». Y concluyen que "las conexiones entre la pobreza y el medio ambiente indican una relación según la cual el fracaso en proteger el medio ambiente puede aumentar la pobreza y el aumento de la pobreza puede conducir al deterioro del medio ambiente». Ernest García aborda explícitamente esta relación en su artículo «Los derechos humanos más allá de los límites al crecimiento", afirmando que «el deterioro del medio ambiente en general, y el cambio climático en particular, afectan negativamente a muchos de los derechos humanos. Concretamente, dificultan la realización de los derechos a la vida, a la salud, a una alimentación adecuada, al acceso a agua potable en condiciones, a la vivienda y a la autonomía personal» (García, 2015, 28).

Un claro reconocimiento -y aprovechamiento- de la vinculación entre Sostenibilidad y DD. HH. ha sido el papel concedido a estos derechos en la elaboración de los Objetivos de Desarrollo Sostenible, aprobados por la Asamblea General de Naciones Unidas, en septiembre de 2015, como guía de la Agenda de Desarrollo 2030 para la transición a la Sostenibilidad. Como puede leerse en el Preámbulo de la resolución aprobada -que lleva por título Transformar nuestro mundo: la Agenda 2030 para el Desarrollo Sostenible-,

Los 17 Objetivos de Desarrollo Sostenible y las 169 metas que anunciamos hoy demuestran la magnitud de esta ambiciosa nueva Agenda universal. [...] También 
se pretende hacer realidad los derechos humanos de todas las personas y alcanzar la igualdad entre los géneros y el empoderamiento de todas las mujeres y niñas. Los Objetivos y las metas son de carácter integrado e indivisible y conjugan las tres dimensiones del Desarrollo Sostenible: económica, social y ambiental.

Es preciso insistir en que esta vinculación entre Sostenibilidad y DD. HH. ha de hacerse aún más explícita e intensa y traducirse en la plena convergencia de las áreas de Educación en Sostenibilidad y Educación en Derechos Humanos, hasta aquí en buena medida inconexas, así como en su aprovechamiento para reforzar la nueva Ciencia de la Sostenibilidad, que abordaremos seguidamente en el último apartado.

\section{La Ciencia de la SOSTENibilidad y por los DeReChos Humanos COMO REVOluCión CIENTÍFICA. PAPEL DE LA EDUCACIÓN}

La Ciencia de la Sostenibilidad surge como nuevo y pujante dominio científico al inicio de este siglo xxi (Kates et al., 2001; Clark, 2007; Kovacevic, 2016) para ayudar a comprender el sistema cada vez más complejo constituido por las sociedades humanas y los sistemas naturales con los que interaccionan y de los que en definitiva forman parte y hacer así posible el tratamiento sistémico, sin reduccionismos ni olvidos, de problemas que, como ya hemos señalado, se potencian mutuamente, por lo que deben ser abordados globalmente (Duarte, 2006; Diamond, 2006). Ese es precisamente uno de los argumentos esgrimidos para justificar la creación en 2006 de una revista específicamente dedicada a la Sustainability Science: "Los problemas a los que la Ciencia de la Sostenibilidad ha de hacer frente no solo son complejos sino que están interconectados. Para encontrar soluciones a los mismos, debemos clarificar primeramente sus relaciones» (Komiyama y Takeuchi, 2006). Este tratamiento global de las interacciones entre sociedad y sistemas naturales impone a la Ciencia de la Sostenibilidad tres características fundamentales que podemos resumir así (Vilches y Gil Pérez, 2015):

- Ha de ser profundamente interdisciplinar, puesto que aborda retos complejos en los que intervienen problemas muy diversos pero estrechamente vinculados, ninguno de los cuales puede ser resuelto aisladamente.

- Se ha comprendido igualmente que para hacer posible la transición a la Sostenibilidad es necesario incorporar a la investigación y toma de decisiones a ciudadanas y ciudadanos que no forman parte del ámbito académico, pero cuyos objetivos, conocimientos y capacidad de intervención resultan imprescindibles para definir y desarrollar estrategias viables (Kovacevic, 2016). Se trata, pues, de una ciencia transdisciplinar.

- Las estrategias concebidas han de responder a una perspectiva amplia, tanto espacial como temporalmente. Ello implica que la perspectiva sea espacialmente "glocal" (a la vez global y local) y que temporalmente contemple tanto el corto plazo como el medio y el largo, esforzándose 
en anticipar posibles riesgos y obstáculos y en aprovechar tendencias positivas.

Planteamientos con esas características permiten evitar las contradicciones que a menudo afectan a medidas adoptadas para resolver problemas puntuales en el tiempo o en el espacio, que olvidan su conexión con otros problemas.

Se ha iniciado así una profunda revolución científica que integra naturaleza y sociedad: después del Heliocentrismo y de la Mecánica Newtoniana, que unificaron Cielo y Tierra, después del Evolucionismo, que estableció el puente entre la especie humana y el resto de los seres vivos, ahora asistimos a la integración del desarrollo social (económico, industrial, educativo, cultural...) con los procesos del denominado mundo natural, buscando comprender las interacciones entre la naturaleza y la sociedad a fin de favorecer a ambas y hacer posible la transición a la Sostenibilidad.

Sin embargo, aunque esta nueva área de conocimiento está teniendo un notable desarrollo (Bettencourt y Kaur, 2011), tras más de tres lustros de existencia, la Ciencia de la Sostenibilidad sigue siendo ignorada, en general, fuera del círculo de quienes contribuyen a su desarrollo como nueva disciplina, lo que viene a limitar su objetivo básico de contribuir a la transición a la Sostenibilidad. Así lo hemos detectado mediante entrevistas realizadas en los campus universitarios a investigadores de distintas áreas, así como mediante análisis bibliográficos de las revistas internacionales (Vilches y Gil Pérez, 2015). Precisamente el hecho de que la Ciencia de la Sostenibilidad se esté conformando como nueva disciplina académica puede explicar un desarrollo relativamente estanco, que se traduce en escasa influencia sobre el resto de la comunidad científica, educativa y movimientos sociales, contradiciendo así los principios de inter- y transdisciplinariedad que están en su origen y limitando su capacidad para acelerar el proceso de transición a la Sostenibilidad, para el que se dispone cada vez de menos tiempo.

Hechos como estos hacen pensar que una profunda revolución científica, capaz de integrar el estudio del desarrollo social y de los procesos naturales, no puede darse únicamente con la creación de una nueva área de conocimiento. Consideramos por ello que la Ciencia de la Sostenibilidad, además de ser una nueva disciplina, ha de constituir una nueva orientación que impregne a las distintas disciplinas: el trabajo de los biólogos, economistas, educadores, físicos, geógrafos, historiadores, ingenieros, químicos, etc., no puede hacerse en compartimentos estancos, sino que ha de tener presente el conjunto de las repercusiones socioambientales de su actividad; y eso obliga a estudiar las aportaciones de las otras disciplinas, así como el punto de vista de los movimientos ciudadanos.

Y esta orientación ha de impregnar igualmente toda la actividad social: la de las corporaciones, sindicatos, medios de comunicación... y, muy particularmente, la actividad política.

En esto ha de consistir la esencia de la Ciencia de la Sostenibilidad, señalábamos en un trabajo reciente (Vilches y Gil Pérez, 2015): las exigencias de interdisciplinariedad, transdisciplinariedad y planteamientos glocales en una perspectiva 
temporal amplia han de impregnar el trabajo de los profesionales de cualquier área, la enseñanza de las distintas disciplinas, la educación ciudadana y, cabe insistir, la acción política que ha de orientar el desarrollo social. No basta con una nueva disciplina, necesitamos un verdadero cambio de paradigma que afecte al conjunto de las actividades sociales, incluida, de forma destacada, la educación, como enfatizan Komiyama y Takeuchi (2006) en el Editorial del primer número de la revista Sustainability Science.

Hoy consideramos necesario añadir a estas características otra igualmente fundamental para la transición a la Sostenibilidad: la consideración explícita de los DD. HH. en la toma de decisiones y, particularmente, en los planteamientos educativos para avanzar hacia sociedades sostenibles. Hablar de repercusiones socioambientales - a corto, medio y largo plazo- exige considerar, prioritariamente, cómo se ven afectados los DD. HH.. Y si bien el papel de la educación ha sido reconocido por los impulsores de la Ciencia de la Sostenibilidad, no hemos encontrado referencias a la educación en DD. HH. Ese es el sentido (más reivindicativo que descriptivo) del título que hemos dado a este apartado, con el que hemos querido hacer explícita la importancia de los DD. HH. en la transición a la Sostenibilidad: "La Ciencia de la Sostenibilidad y por los Derechos Humanos como revolución científica». Una revolución científica a la que la educación puede y debe contribuir, planteada y formulada conjuntamente como Educación en Sostenibilidad y en Derechos Humanos o, más explícitamente, Educación para la Sostenibilidad y la universalización de los Derechos Humanos.

\section{REFERENCIAS BIBLIOGRÁFICAS}

Almuiña, C. (2016) La información veraz como derecho de tercera generación, en PANDO Ballesteros, M. ${ }^{a}$ P.; Muñoz Ramírez, A. y Garrido Rodríguez, P. (dirs.) Pasado y presente de los derechos humanos. Mirando al futuro. Madrid, Catarata, 169-189.

Aznar, P.; Martínez-Agut, M. P.; Palacios, B.; Piñero, A. y Ull, M. A. (2011) Introducing sustainability into university curricula: an indicator and baseline survey of the views of university teachers at the University of Valencia. Environmental Education Research, 17 (2), 145-166.

AZnar, P. y Ull, M. A. (2009) La formación de competencias básicas para el Desarrollo Sostenible: el papel de la Universidad. Revista de Educación, número extraordinario, 219-237.

BARRón Ruiz, A.; NAVARrete, A. y Ferrer, D. (2010) Sostenibilización curricular en las Universidades españolas. ¿Ha llegado la hora de actuar? Revista Eureka sobre Enseñanza y Divulgación de las Ciencias, 7, número extraordinario, 388-399.

Bermejo, R. (2011) Manual para una economía sostenible. Madrid, Catarata.

BetTencourt, L. y KaUR, J. (2011) Evolution and structure of sustainability science. Proceedings of the National Academy of Sciences (PNAS), 108 (49), 19540-19545.

BybeE, R. W. (1991) Planet Earth in crisis: how should science educators respond? The American Biology Teacher, 53 (3), 146-153.

Clark, W. C. (2007) Sustainability Science: A room of its own. Proceedings of the National Academy of Sciences (PNAS), 104 (6), 1737-1738. 
Comisión Mundial del Medio Ambiente y del Desarrollo (1988) Nuestro Futuro Común. Madrid, Alianza.

Cortina, A. y Pereira, G. (eds.) (2009) Pobreza y libertad. Erradicar la pobreza desde el enfoque de Amartya Sen. Madrid, Tecnos.

De la Rosa, C. (2015) La disputa de los Derechos Humanos. A modo de Introducción, en vV. AA. Más allá de lo imposible. La dimensión política de los derechos humanos en el siglo XXI. Tafalla, Txalaparta, 9-20.

Diamond, J. (2006) Colapso. Barcelona, Debate.

Disterheft, A.; Caeiro, S.; Azeiteiro, U. M. y Leal Filho, W. (2013) Sustainability Science and Education for Sustainable Development in Universities: A Way for Transition, en Caeiro, S.; Leal Filho, W.; Jabbour, Ch. y Azeiteiro, U. M. (eds.) Sustainability Assessment Tools in Higher Education Institutions. Cham, Springer, 3-27.

DuARTE, C. (coord.) (2006) Cambio Global. Impacto de la actividad humana sobre el sistema Tierra. Madrid, CSIC.

Engelman, R. (2013) Más allá de la sosteniblablá, en THe Worldwatch InstituTe. La situación del mundo 2013. ¿Es aún posible lograr la Sostenibilidad? Barcelona, Icaria.

FelBer, C. (2012) La economía del bien común. Barcelona, Deusto.

Flavin, C. (2008) Construir una economía baja en carbono, en Worldwatch InstiTuTE La situación del mundo 2008. Innovaciones para una economía sostenible. Barcelona, Icaria, capítulo 6.

Folch, R. (1998) Ambiente, emoción y ética. Barcelona, Ariel.

FOLKE, C. (2013) Respetar los límites del planeta y recuperar la conexión con la biosfera, en THE Worldwatch Institute La situación del mundo 2013. ¿Es aún posible lograr la Sostenibilidad? Barcelona, Icaria.

Gaigher Bósio Campello, L. y Coimbra de Carvalho, L. (2016) Direitos Humanos e Meio Ambiente: potencializando duas expressões em um link, en PANDO BALlesteros, M. ${ }^{a}$ P.; Muñoz Ramírez, A. y Garrido Rodríguez, P. (dirs.) Pasado y presente de los derechos bumanos. Mirando al futuro. Madrid, Catarata, 625-638.

García, E. (2004) Medio ambiente y sociedad. La civilización industrial y los límites del planeta. Madrid, Alianza Editorial.

García, E. (2015) Los derechos humanos más allá de los límites al crecimiento. Ambienta, $113,28-41$.

GarCía PÉREZ, F. F. (2015) Educar en la escuela para afrontar los problemas del mundo, en VV. AA. Más allá de lo imposible. La dimensión política de los derechos humanos en el siglo XXI. Tafalla, Txalaparta, 145-171.

Gil PÉREZ, D. y Vilches, A. (2005) ¿Qué desafíos tiene planteados hoy la humanidad? Educación para el Desarrollo Sostenible, en GiL-PÉrez, D.; MACEdo, B.; MARTínez Torregrosa, J.; SifRedo, C.; VAldés, P. y Vilches, A. (eds.) ¿Cómo promover el interés por la cultura científica? Una propuesta didáctica fundamentada para la educación científica de jóvenes de 15 a 18 años. Santiago, OREALC/UNESCO, 297-326.

IzQuierdo BEnito, M. ${ }^{a}$ J. (2015) Cambios estructurales para la realización efectiva de los Derechos Humanos para las mujeres, en vV. AA. Más allá de lo imposible. La dimensión politica de los derechos humanos en el siglo XXI. Tafalla, Txalaparta, 41-61.

Kates, R. W.; Clark, W. C.; Corell, R.; Hall, J. M.; Jaeger, C. C.; Lowe, I.; McCarthy, J. J.; Schellnhuber, H. J.; Bolin, B.; Dickson, N. M.; FaucheuX, S.; Gallopin, G. C.; GrÜBler, A.; Huntley, B.; Jäger, J.; Jodha, N. S.; Kasperson, R. E.; Mabogunje, A.; Matson, 
P.; Mooney, H.; Moore, B.; O'Riordan, T. y Svedin, U. (2001) Sustainability Science. Science, 292 (5517), 641-642.

KLeIn, N. (2015) Esto lo cambia todo. El capitalismo contra el clima. Barcelona, Paidós.

KomiYama, H. y TaKeuchi, K. (2006) Sustainability science: building a new discipline. Sustainability Science, 1 (1), 1-6.

KovaceVIC, M. (2016) Mobilising for sustainability. Celebrating and strengthening stories of connection. Future Earth's newsletter, October 2016 (de libre acceso en Internet).

MARTínez QuinTEIRO, M. E. (2016) El discurso de los derechos humanos en perspectiva histórica. El síndrome de la torre de Babel, en Pando Ballesteros, M. ${ }^{a}$ P.; MuÑoz Ramírez, A. y Garrido Rodríguez, P. (dirs.) Pasado y presente de los derechos humanos. Mirando al futuro. Madrid, Catarata, 41-59.

Mayor Zaragoza, F. (2000) Un mundo nuevo. Barcelona, unesCo-Círculo de lectores.

Meadows, D. H.; Meadows, D. L.; Randers, J. y Behrens, W. (1972) Los límites del crecimiento. Madrid, Fondo de Cultura Económica.

NARedo, J. M. (1998) Sobre el rumbo del mundo, en SÁnchez Ron, J. M. (dir.) Pensamiento Crítico vs. Pensamiento único. Madrid, Debate.

Nascimento, J. (2016) Os princípios dos Direitos Humanos, en Pando Ballesteros, M. ${ }^{a}$ P.; Muñoz Ramírez, A. y Garrido Rodríguez, P. (dirs.) Pasado y presente de los derechos bumanos. Mirando al futuro. Madrid, Catarata, 99-102.

Novo, M. (2006) El desarrollo sostenible. Su dimensión ambiental y educativa. Madrid, UNESCO-Pearson.

Novo, M. y Murga-Menoyo, M. Á. (2015) The Processes of Integrating Sustainability in Higher Education Curricula: A Theoretical-Practical Experience Regarding Key Competences and Their Cross-Curricular Incorporation into Degree Courses, en LEAL FILHO, W. (ed.) Transformative Approaches to Sustainable Development at Universities. Cham, Springer, 119-133.

Pando Ballesteros, M. ${ }^{a}$ P (2016a) Educación en Derechos Humanos. La Aportación de la Universidad de Salamanca, en Pando Ballesteros, M. ${ }^{a}$ P.; MuÑOZ Ramírez, A. y Garrido Rodríguez, P. (dirs.) Pasado y presente de los derechos humanos. Mirando al futuro. Madrid, Catarata, 65-81.

Pando Ballesteros, M. ${ }^{a}$ P. (2016b) Introducción, en Pando Ballesteros, M. ${ }^{a}$ P.; MuÑOz Ramírez, A. y Garrido Rodríguez, P. (dirs.) Pasado y presente de los derechos humanos. Mirando al futuro. Madrid, Catarata, 15-37.

PeArCE, F. (2007) La última generación. Benasque, Barrabes.

RifKin, J. (2010) La Civilización Empática. Barcelona, Paidós.

SACHS, J. (2008) Economía para un planeta abarrotado. Barcelona, Debate.

SAn Segundo, R. (2016) Nuevas formas de lectura. Conocimiento y ciudadanía en el entorno digital, en Pando Ballesteros, M. ${ }^{a}$ P.; MuÑoz Ramírez, A. y Garrido Rodríguez, P. (dirs). Pasado y presente de los derechos humanos. Mirando al futuro. Madrid: Catarata, 651-666.

Sen, A. (2000) Desarrollo y libertad. Barcelona, Planeta.

Tilbury, D. (1995) Environmental education for sustainability: defining the new focus of environmental education in the 1990s. Environmental Education Research, 1 (2), $195-212$.

THOMAS, I. (2004) Sustainability in tertiary curricula: what is stopping it happening? International Journal of Sustainability in Higher Education, 5 (1), 33-47. 
unESCO (2014) Hoja de ruta para la ejecución del programa de acción mundial de Educación para el Desarrollo Sostenible. [Publicación disponible en acceso abierto. Título original: UNESCO Roadmap for Implementing the Global Action Programme on Education for Sustainable Development].

Vercher, A. (1998) Derechos humanos y medio ambiente. Claves de Razón Práctica, 84, $14-21$

Vilches, A. y Gil PÉrez, D. (2003) Construyamos un futuro sostenible. Diálogos de supervivencia. Madrid, Cambridge University Press.

Vilches, A. y Gil-PÉrez, D. (2015) Ciencia de la Sostenibilidad: ¿Una nueva disciplina o un nuevo enfoque para todas las disciplinas? Revista Iberoamericana de Educación, 69 (1), 39-60.

Vilches, A.; Macías, O. y Gil-PÉRez, D. (2014) La transición a la Sostenibilidad. Un desafío urgente para la ciencia, la educación y la acción ciudadana. Temas clave de reflexión y acción. Madrid, OEI. ISBN 978-84-7666-204-5.

WORLDWATCh InSTITUTE (1984-2016) The State of the World. New York, W. W. Norton. [Versiones en castellano: La situación del mundo. Barcelona, Icaria].

WwF (2016) Living Planet Report 2016. Risk and resilience in a new era. Gland, wwF International. ISBN 978-2-940529-40-7. [Resumen en castellano: Informe planeta vivo 2016: Riesgo y Resiliencia en una nueva era]. 